\title{
Avaliação da Acidose Metabólica em Pacientes Graves: Método de Stewart-Fencl-Figge Versus a Abordagem Tradicional de Henderson-Hasselbalch*
}

\author{
Assessment of Metabolic Acidosis in Critically ill Patients: \\ Method of Stewart-Fencl-Figge Versus the \\ Traditional Henderson-Hasselbalch Approach.
}

Manuela Borges Gavaza Barbosa ${ }^{1}$, Crésio de Aragão Dantas Alves², Hélio Queiroz Filho

\section{RESUMO}

JUSTIFICATIVA E OBJETIVOS: Rever estratégias de avaliação da acidose metabólica dando ênfase ao método de Stewart-Fencl-Figge versus a abordagem tradicional de Henderson-Hasselbalch.

CONTEÚDO: A acidose metabólica é um distúrbio comum em pacientes criticamente enfermos, sendo importante causa de depressão da função miocárdica e sensível indicador de má perfusão tissular. Tradicionalmente, é avaliada através do método de Henderson-Hasselbalch no qual a gasometria arterial fornece informações sobre a existência e o tipo de distúrbio ácido-básico. Porém, nem sempre, esse método é capaz de explicar os seus mecanismos causais e,

1. Intensivista Pediátrica do Hospital Santo Antônio, Obras Sociais Irmã Dulce e do Hospital Salvador, Salvador, BA.

2. Professor de Pediatria da Faculdade de Medicina da Universidade Federal da Bahia - UFBa; Coordenador da Residência em Endocrinologia Pediátrica, Hospital Universitário Professor Edgard Santos - UFBa.

3. Mestre em Medicina pela Universidade Federal da Bahia; Chefe da Unidade de Terapia Intensiva Pediátrica do Hospital Salvador.

*Recebido do Hospital Santo Antônio, Obras Sociais Irmã Dulce, Salvador, BA.

- Parte da Monografia apresentada pela Dra. Manuela Borges Gavaza Barbosa, à AMIB, em 2005, como Pré-Requisito para Obtenção do Título de Especialista em Terapia Intensiva Pediátrica.

Apresentado em 02 de agosto de 2006

Aceito para publicação em 01 de dezembro de 2006

Endereço para correspondência:

Dra. Manuela Borges Gavaza Barbosa

Rua Bombeiro Eliezer de Alexandrino, 107/102.

41710-790 Salvador, BA

Fones: (71) 3362-1755 - 8118-7973

E-mail: manuborges@hotmail.com

(C)Associação de Medicina Intensiva Brasileira, 2006 por isso, muitos estudos têm sido feitos na tentativa de melhorar sua interpretação. O método de StewartFencl-Figge, calculado através de fórmula matemática, em que além da gasometria arterial, são utilizados níveis séricos de vários eletrólitos, lactato e albumina, nos fornece informações mais fidedignas permitindo detectar anormalidades metabólicas mistas e estimar a magnitude de cada componente, principalmente na presença de múltiplas disfunções orgânicas. Nesses pacientes, a presença de ânions não mensurados no plasma é importante mecanismo de acidose metabólica e sua detecção precoce é fundamental para se evitar efeitos deletérios sobre o organismo.

CONCLUSÕES: A abordagem tradicional de Henderson-Hasselbalch falha em analisar os mecanismos da acidose metabólica e possui muitas variáveis que interferem no seu resultado, principalmente no paciente criticamente enfermo. O método de Stewart-Fencl-Figge proporciona abordagem mais completa para avaliação da acidose metabólica, sugerindo seus mecanismos e orientando a terapêutica. Como alternativa, o anion gap corrigido pela albumina e lactato parece ser tão eficiente em identificar a presença de anions não mensurados quanto o método de Stewart.

Unitermos: acidose metabólica; distúrbio ácido-básico; equação de Henderson-Hasselbalch, equações de Fencl, fórmulas de Figge, método de Stewart

\section{SUMMARY}

BACKGROUND AND OBJECTIVES: To review strategies of assessment of metabolic acidosis giving emphasis to the of Stewart-Fencl-Figge method versus the traditional method of Henderson-Hasselbalch.

CONTENTS: Metabolic acidosis is a common issue in critically ill patients, an important cause of myocardial 
contractility depression and sensible marker of impaired tissue oxygenation. Traditionally, is evaluated by the Henderson-Hasselbalch approach in which an arterial blood sample provides information about the presence and type of acid base disturbance. However, this method is not always capable to explain the causes of the metabolic acidosis and, therefore, several studies have explored mechanisms to improve its interpretation. The Stewart-Fencl-Figge method calculated through a mathematical formula, where in addition to arterial blood gas levels, serum levels of electrolytes, lactate and albumin are used, supplies trustworthy information allowing detection of mixed metabolic abnormalities and quantification of the magnitude of each component, mainly in patients with multiple organic dysfunctions. In these individuals, the presence of unmeasured anions in the plasma is an important mechanism of metabolic acidosis and its early detection fundamental to avoid deleterious effect on the organism.

CONCLUSIONS: The traditional Henderson-Hasselbalch approach fails in analyzing the underlying mechanisms of metabolic acidosis and possesses many variables that intervene with its result especially in the critically ill patient. The Stewart-Fencl-Figge method offers a broader analysis of metabolic acidosis, indicating its mechanisms and guiding a better therapeutically strategy. As an alternative, the albumin-corrected and lactate-corrected anion gap seems to be as useful as the Stewart approach in identifying the unmeasured anions.

Key Words: acid base disturbance, equation, Fencl's equations, Figge's formulas, Henderson-Hasselbalch's, metabolic acidosis, Stewart's method

\section{INTRODUÇÃO}

Os distúrbios do equilíbrio ácido-básico são comuns em pacientes criticamente enfermos. Freqüentemente, a presença desses distúrbios indica uma doença subjacente grave e, no caso de acidose metabólica, é um indicador de evolução clínica desfavorável ${ }^{1}$.

A acidose metabólica é caracterizada por redução do pH sanguíneo como resultado do acúmulo de ácidos não voláteis ou perda de bicarbonato sérico ${ }^{2,3}$. Sua fisiopatologia é extremamente complexa, principalmente nos pacientes com sepse, insuficiência hepática e trauma ${ }^{4}$.

A persistência da acidose após as medidas terapêuticas iniciais é sinal de mau prognóstico ${ }^{5}$. A gravidade e reversibilidade da disfunção, no entanto, dependem da doença de base e da magnitude da desordem. Apesar de existirem muitas informações sobre os efeitos da acidose metabólica em células isoladas, na perfusão dos órgãos e na imunidade, esses efeitos ainda são pouco compreendidos quando se trata dos seus aspectos clínicos ${ }^{6}$. Isto é especialmente importante nos pacientes criticamente enfermos pela dificuldade em separar os efeitos da acidose dos efeitos da doença de base ${ }^{1,3}$.

Tradicionalmente, os distúrbios ácido-básicos são explicados através do sistema ácido carbônico-bicarbonato. Essa relação é descrita através da equação de Henderson-Hasselbalch ${ }^{7}$. Porém, este método, falha em explicar os prováveis mecanismos causais nos extremos dos distúrbios fisiológicos ${ }^{8}$. Por isso, diversos estudos têm sido feitos na tentativa de encontrar novos métodos para aprimorar a interpretação dos distúrbios ácido-básicos. O cálculo da diferença de íons fortes é um desses métodos ${ }^{8}$.

Este artigo teve por objetivo rever métodos de avaliação da acidose metabólica dando ênfase ao método de Stewart-Fencl-Figge versus a abordagem tradicional de Henderson-Hasselbalch.

\section{MÉTODO DE HENDERSON-HASSELBALCH}

Há mais de um século Henderson utilizou a teoria das espécies de carbono para propor o balanço ácido-básico no sangue ${ }^{9}$. Em seguida, Hasselbalch criou uma fórmula simples para descrever este equilíbrio ${ }^{9}$. Desde então, a equação de Henderson-Hasselbalch e o excesso de base são utilizados para descrever os distúrbios do equilíbrio ácido-básico no sangue ${ }^{10}$. Apesar de suas limitações, este método continua sendo amplamente utilizado, por sua fácil aplicação na análise da acidose metabólica, principalmente em pacientes que não estão com múltiplas disfunções orgânicas ${ }^{9,10}$.

A avaliação clássica utiliza os seguintes parâmetros: $\mathrm{pH}, \mathrm{PaCO}_{2}$, bicarbonato, excesso de base $(\mathrm{BE}=$ Base Excess) e anion gap (AG) ou intervalo aniônico.

- pH: é o logaritmo negativo da concentração de íons hidrogênio livre sendo definido pela razão entre a $\mathrm{PaCO}_{2}$ e o bicarbonato plasmático. Seu valor normal situa-se entre 7,35 e 7,43 ${ }^{11}$. O pH é calculado pelas fórmulas:

$\mathrm{pH}=\left[6,10+\log \left(\mathrm{HCO}_{3}^{-} / \mathrm{PaCO}_{2} \times 0,030\right)\right]$

$\mathrm{pH}=-\log \left(\mathrm{H}^{+}\right)=-\log \left(3,98 \times 10^{-8}\right)=-(0,60-8,0)=7,4$ - $\mathrm{PaCO}_{2:}$ representa a pressão parcial de $\mathrm{CO}_{2}$ no sangue arterial e é regulada pela ventilação pulmonar. Seus valores normais variam entre 35 e $45 \mathrm{mmHg}^{11}$. 
- Excesso de base (BE): descrito em $\mathrm{mEq} / \mathrm{L}$, é obtido pela multiplicação do desvio do bicarbonato a partir do valor médio de $22,9 \mathrm{mEq} / \mathrm{L}$ e por fator igual a 1,2. Um valor inferior a $-5 \mathrm{mEq} / \mathrm{L}$ é indicativo de acidose metabólica ${ }^{9,12}$. Um problema associado ao cálculo do $B E$ é que ele varia com alterações dos eletrólitos e da albumina. Um decréscimo de $1 \mathrm{~g} / \mathrm{dL}$ na albumina sérica diminui em $3,7 \mathrm{mEq} / \mathrm{L}$ o excesso de base. Outra limitação é que o excesso de base não define se a acidose é causada pelo lactato, cetoácidos, hipercloremia ou uma combinação desses. Ele pode ser calculado através da seguinte fórmula:

$\mathrm{BE}=0,9287 \times[(\mathrm{BIC}-24,4)]+(14,83 \times \mathrm{pH}-7,4)]$

- Anion gap (AG): descrito em $\mathrm{mEq} / \mathrm{L}$, é utilizado para a medida de ânions não mensurados no plasma. Seus valores normais situam-se entre $12 \pm 2 \mathrm{mEq} / \mathrm{L}$. Valores superiores a $16 \mathrm{mEq} / \mathrm{L}$ indicam a presença de ânions não mensuráveis no plasma. Esse método sofre interferência em casos de hipoalbuminemia e alterações no fósforo ${ }^{13}$. A fórmula usada para seu cálculo é:

$A G=\left[\left(\mathrm{Na}^{+}+\mathrm{K}^{+}\right)-\left(\mathrm{Cl}^{-}+\right.\right.$Bicarbonato $\left.)\right]$

- Anion gap corrigido ( $A G c)$ : é o cálculo do AG corrigido pela albumina e pelo lactato, que estão quase sempre alterados (hipoalbuminemia e hiperlactatemia) em pacientes criticamente enfermos ${ }^{14,15}$.

$A G c=A G+0,25 \times(40-$ [albumina] $)-$ lactato.

\section{MÉTODO DE STEWART-FENCL-FIGGE}

Em 1981, Peter Stewart, físico canadense, observou que embora o excesso de base fornecesse a magnitude do distúrbio ácido-básico, o modelo tradicional não oferecia informações sobre o mecanismo da disfunção ${ }^{13,16,18}$. Então, baseado na lei da conservação das massas e cargas (eletro-neutralidade), criou um complexo matemático de fórmulas para descrever o balanço ácido-básico ${ }^{17}$. Com base nesses princípios, Figge e col., definiram a relação entre fosfato, albumina plasmática e íons hidrogênio, concluindo que a albumina é o maior contribuinte dos ácidos fracos ${ }^{14,19}$. De acordo com essa teoria, três variáveis independentes determinam a concentração do íon hidrogênio: diferença de íons fortes (SID = strong ion difference), concentração total de ácidos fracos não voláteis, principalmente albumina e fosfato, e pressão parcial de gás carbônico $\left(\mathrm{PaCO}_{2}\right)^{13}$. Os princípios e fórmulas básicas utilizadas pelo método de Stewart são:

- Diferença de íons forte aparente (SIDa): é a diferença entre os cátions abundantes no organismo $\left(\mathrm{Na}^{+}, \mathrm{K}^{+}\right.$, $\mathrm{Mg}^{2+}$ e $\left.\mathrm{Ca}^{2+}\right)$ e os ânions $\left(\mathrm{Cl}^{-} \text {, lactato }\right)^{20-22}$. Esse SID é chamado de aparente porque não leva em consideração ácidos fracos como albumina e fosfato, que também podem estar presentes influenciando o valor do SID. Ele é utilizado na fórmula de Stewart para calcular o SIG (strong íon gap). A fórmula para o cálculo do $\mathrm{SIDa}$ (todas concentrações em $\mathrm{mEq} / \mathrm{L}$ ), cujo valor normal varia entre 40 e $42 \mathrm{mEq} / \mathrm{L}$ é:

$\mathrm{SIDa}=\left[\left(\mathrm{Na}^{+}+\mathrm{K}^{+}+\mathrm{Mg}^{2+}+\mathrm{Ca}^{2+}\right)-\left(\mathrm{Cl}^{-}+\right.\right.$lactato $\left.)\right]$

- Diferença de íons forte efetiva (SIDe): é o SIDa que leva em consideração a participação dos ácidos fracos no equilíbrio elétrico. Seu valor normal é $-40 \mathrm{mEq} / \mathrm{L}$. Figge e col. baseados nos princípios de Stewart, desenvolveram uma equação que calcula o SIDe através da seguinte fórmula:

$\mathrm{SIDe}=\left[2,46 \times 10^{-8} \times \mathrm{PaCO}_{2}(\mathrm{mmHg}) / 10^{-\mathrm{pH}}+(\right.$ Albumina $(\mathrm{g} / \mathrm{dL}) \times(0,123 \times \mathrm{pH}-0,631)+($ Fosfato $(\mathrm{mg} / \mathrm{dL}) \times(0,309$ $x \mathrm{pH}-0,469)]$.

- Gap da diferença dos ion fortes (SIG): é a diferença entre o SIDa e o SIDe. Seu valor normal em pacientes sadios varia de 0 a $2 \mathrm{mEq} / \mathrm{L}$. Quando o SIG é maior que 2, indica que os ânions não mensurados excederam os cátions, e quando menor que zero sugere que cátions excederam os ânions ${ }^{22}$. Estudos mais recentes demonstraram que um SIG $>2 \mathrm{mEq} / \mathrm{L}$ está independentemente associado a mortalidade em pacientes criticamente enfermos com acidose metabólica ${ }^{6}$.

\section{DISCUSSÃO}

A presença de acidose metabólica é utilizada como importante indicador de baixo débito cardíaco e má perfusão tissular. Uma série de estudos mostrou que os níveis séricos de lactato estão significativamente elevados nesses pacientes ${ }^{1,23}$. Embora em adultos criticamente enfermos, o lactato corresponda à metade das alterações na acidose metabólica ${ }^{1}$, em pacientes pediátricos acidose metabólica por excesso de lactato ocorre em apenas $10 \%$ das crianças, sendo os ânions não mensuráveis os maiores contribuintes deste distúrbio ${ }^{1}$. Em alguns estudos, o aumento inicial do lactato nas primeiras 24 horas mostrou ser superior ao excesso de base em relação à predição de morbimortalidade ${ }^{23}$.

Por outro lado, o excesso de base e o anion gap, utilizados nos métodos tradicionais de análise dos distúrbios ácido-básicos, freqüentemente falham em detectar ânions não mensurados como fator causador da acidose metabólica. Quanto maior as anormalidades plasmáticas do sódio, cloro e albumina, alterações freqüentes em pacientes graves, maiores serão as dife- 
renças entre o excesso de base (método tradicional) e a diferença de ácidos fortes (método de Stewart). Além disso, os ânions não mensuráveis identificados pelo SIG estão mais relacionados à morbimortalidade ${ }^{25}$.

Confirmando essas suposições, Balasubramanyan e col., compararam três métodos de identificação de ânions não mensuráveis em pacientes pediátricos criticamente enfermos: excesso de base, anion gap e o gap da diferença de íons fortes $(\mathrm{SIG})^{25}$. A presença de ânions não mensuráveis foi fracamente identificada pelo excesso de base ou anion gap. A concentração sérica do lactato correlacionou-se melhor com o SIG do que com o excesso de base ou anion gap. O aumento de ânions não mensurados identificados pelo SIG estava fortemente associado à mortalidade do que o excesso de base, anion gap ou lactato sérico ${ }^{25}$. Entretanto, Moviat e col. utilizando o anion gap corrigido pela albumina e lactato mostraram boa correlação entre esse parâmetro e o SIG na identificação de anions não mensuráveis em pacientes internados na UTI com acidose metabólica, sugerindo que tal abordagem poderia substituir o método de Stewart quando da avaliação da acidose metabólica em pacientes criticamente enfermos ${ }^{15}$. Isto confirmou a limitação do anion gap não corrigido na identificação de anions não mensurados na causalidade da acidose metabólica ${ }^{26}$.

Na prática clínica, o método de Stewart-Fencl-Figge é útil quando se deseja uma análise mais fidedigna da acidose metabólica principalmente na presença de alterações nos níveis de albumina, fosfato, lactato, cetoácidos ${ }^{11}$.

O quadro 1 apresenta as principais vantagens e desvantagens dos dois métodos de avaliação da acidose metabólica.

\section{CONCLUSÃO}

Os métodos tradicionais de avaliação da acidose metabólica continuam sendo muito importantes principalmente naqueles pacientes que possuem poucas variáveis que interfiram em seu resultado. Entretanto, pacientes criticamente enfermos apresentam doenças mais complexas, com múltiplos distúrbios e muitas variáveis que interferem na análise da acidose metabólica quando são utilizados os métodos tradicionais. Nesses casos, os distúrbios do equilíbrio ácido-básico são mais fáceis de entender, explicar e racionalizar quando se utiliza o método de Stewart. Como suas variáveis são matematicamente válidas isto provê maior utilização clínica do que parâmetros como excesso de base e anion gap. Por estas razões, o método de Stewart, não muito utilizado na prática clínica por ser uma equação matemática complexa, vem ganhando popularidade nas unidades de terapia intensiva. O problema das equações matemáticas pode ser facilmente resolvido através de programas de computadores. Com isto, seu uso poderá ser instituído permitindo melhor avaliação dos pacientes com distúrbios ácido-básicos e múltiplas disfunções orgânicas. Recentemente, tem-se mostrado que o método tradicional associado ao AG corrigido pela albumina e pelo lactato é tão eficiente em identificar a presença de anions não mensurados no paciente criticamente enfermo quanto o método de Stewart. Mais estudos serão necessários para identificar quais são os anions não mensurados (p. ex.: compostos orgânicos e inorgânicos, salicilatos, penicilina) e qual sua participação na patogênese da acidose metabólica em pacientes criticamente enfermos.

Quadro 1 - Comparação entre Dois Métodos de Avaliação da Acidose Metabólica

\begin{tabular}{|c|c|c|}
\hline Métodos & Vantagens & Desvantagens \\
\hline Henderson-Hasselbalch & $\begin{array}{l}\text { Fórmula simples de fácil aplicação. } \\
\text { Útil em pacientes que não apresentem múltiplas disfun- } \\
\text { ções orgânicas. } \\
\text { Necessita apenas da gasometria arterial e do } \mathrm{Na}^{+}, \mathrm{K}^{+} \text {e } \\
\mathrm{Cl}^{-} \text {para calcular o anion gap. }\end{array}$ & $\begin{array}{l}\text { Não explica os mecanismos causais nos extre- } \\
\text { mos dos distúrbios fisiológicos. } \\
\text { Sofre interferências quando há variações nos } \\
\text { valores de albumina e eletrólitos. } \\
\text { O excesso de base e o anion gap freqüentemen- } \\
\text { te falham em detectar ânions não mensurados } \\
\text { como causadores da acidose metabólica. }\end{array}$ \\
\hline
\end{tabular}




\section{REFERÊNCIAS}

01. Murray DM, Olhsson V, Fraser JI - Defining acidosis in postoperative cardiac patients using Stewart's method of strong ion difference. Pediatr Crit Care Med, 2004;5:240-245.

02. Levraut J, Grimaud D - Treatment of metabolic acidosis. Curr Opin Crit Care, 2003;9:260-265

03. Nogueira PCK - Acidose Metabólica, em: Matsumoto T, Carvalho WB, Hirschheimer MR - Terapia Intensiva Pediátrica. São Paulo: Atheneu. 1997;578-582.

04. Gauthier PM, Szerlip HM - Metabolic acidosis in the intensive care unit. Crit Care Clin, 2002;18:289-308.

05. Bruno F, Santana JCB, Lago P Distúrbios Hidroeletrolíticos na Criança, em: Piva JP, Garcia PCR - Medicina Intensiva em Pediatria. Rio de Janeiro: Revinter. 2005;322-323.

06. Gunnerson KJ, Saul M, He S et al - Lactate versus non-lactate metabolic acidosis: a retrospective outcome evaluation of critically ill patients. Crit Care, 2006;10:R22-R30.

07. Boyle M, Lawrence $\mathrm{J}$ - An easy method of mentally estimating the metabolic component of acid/base balance using the Fencl-Stewart approach. Anaesth Intensive Care, 2003;31:538-547.

08. Kellum JA - Metabolic acidosis in the critically ill: lessons from physical chemistry. Kidney Int, 1998;53:(Suppl66):S81-S86.

09. Corey HE - Stewart and beyond: new models of acid-base balance. Kidney Int, 2003;64:777-787.

10. Rocco JR - Diagnóstico dos distúrbios do metabolismo ácido-base. RBTI, 2003;15:184-192.

11. Adelman RD, Solhaug MJ - Fisiopatologia dos Líquidos Corporais e Terapia de Hidratação, em: Behrman RE, Kliegman RM, Jenson HB Medicina Intensiva em Pediatria. Rio de Janeiro: Guanabara Koogan, 2002;203-207.

12. Schlichitig R, Grogono AW, Severinghaus JW - Human $\mathrm{PaCO}_{2}$ and standard base excess compensation for acid-base imbalance. Crit Care Med, 1998;26:1173-1179.
13. Constable PD - Clinical assessment of acid-base status. Strong ion difference theory. Vet Clin North Am Food Anim Pract, 1999;15:447-471.

14. Hatherill M, Waggie Z, Purves $L$ et al - Correction of the anion gap for albumin in order to detect occult tissues anions in shock. Arch Dis Child, 2002;87:526-529.

15. Moviat $\mathrm{M}$, van Haren $\mathrm{F}$, van der Hoeven $\mathrm{H}$ - Conventional or physicochemical approach in intensive care unit patients with metabolic acidosis. Crit Care, 2003;7:R41-R45

16. Stewart PA - Modern quantitative acid-base chemistry. Can J Physiol Pharmacol, 1983;61:1444-1461.

17. Story DA, Morimatsu H, Bellomo R - Strong ions, weak acids and base excess: a simplified Fencl-Stewart approach to clinical acid-base disorders. Br J Anaesth, 2004;92:54-60.

18. Fencl V, Leith DE - Stewart's quantitative acid-base chemistry: applications in biology and medicine. Respir Physiol, 1993;91:1-16.

19. Durward A, Mayer A, Skellett S et al - Hypoalbuminaemia in critically ill children: incidence, prognosis, and influence on the anion gap. Arch Dis Child, 2003;88:419-422.

20. Dorje P, Adhikary G, McLaren ID et al - Dilutional acidosis or altered strong ion difference. Anesthesiology, 1997;87:1011-1012.

21. Whitehair KJ, Haskins SC, Whitehair JG et al - Clinical applications of quantitative acid-base chemistry. J Vet Intern Med, 1995;9:1-11.

22. Kellum JA, Kramer DJ, Pinsky MR - Strong ion gap: a methodology for exploring unexplained anions. J Crit Care, 1995;10:51-55.

23. Husain FA, Martin MJ, Mullenix PS et al - Serum lactate and base deficit as predictors of mortality and morbidity. Am J Surg, 2003;185:485-491.

24. Rocktaeschel J, Morimatsu H, Uchino S et al - Unmeasured anions in critically ill patients: can they predict mortality? Crit Care Med, 2003;31:2131-2136.

25. Balasubramanyan Napa, Havens PL, Hoffman GM - Unmeasured anions identified by the Fencl-Stewart method predict mortality better than base excess, anion gap, and lactate in patients in the pediatric intensive care unit. Crit Care Med, 1999;27:1577-1581.

26. Kellum JA - Closing the gap on unmeasured anions. Crit Care, 2003;7:219-220. 